\title{
The dynamics of a neoclassical tearing mode (NTM) influenced by
}

\section{energetic ions on EAST}

Erzhong $\mathrm{Li}^{1}$, V. Igochine ${ }^{2}$, L. Xu ${ }^{1}$, T. Shi ${ }^{1}, \mathrm{H} . \mathrm{Zhao}^{1}$, Y. Liu ${ }^{1}, \mathrm{~A} . \mathrm{Ti}^{1}$, R. White ${ }^{3}$, J. Zhang ${ }^{1}$, Y. Zhu ${ }^{4}$, J. Huang ${ }^{1}$, B. Shen ${ }^{1}$, S. Lin $^{1}$, J. Qian ${ }^{1}$, X. Gong $^{1}, \mathrm{~L} . \mathrm{Hu}^{1}$, and EAST Contributors

1 Institute of Plasma Physics, Chinese Academy of Science, Hefei 230031, People's Republic of China

2 MPI fur Plasmaphysik, D-85748 Garching, Germany

3 Plasma Physics Laboratory, Princeton University, P.O. Box 451, Princeton, NJ 08543, USA

4 University of California-Irvine, Irvine, California 92697-4575, USA

$$
\text { E-mail: } \underline{\text { zhonglee@ipp.ac.cn }}
$$

\begin{abstract}
In the 2014 year's campaign of Experimental Advanced Superconducting Tokamak (EAST), a series of Magnetohydrodynamics (MHD) instabilities were observed as the launching of Neutral Beam Injection (NBI), the most interesting one of which is the neoclassical tearing mode (NTM). Evidence clearly shows that a kink mode present after a strong sawtooth-like (ST-like) crash leaves a perturbation near the location of the magnetic island, providing the initial seed. The interaction of energetic ions makes the magnetic island oscillate both in island width and in rotation frequency. Analysis indicates that the bulk plasma still
\end{abstract}


dominates the dynamics of NTM, and the orbit excursion of energetic ions induces a polarization current and modifies the width and rotation frequency of the neoclassical magnetic island.

Keywords: neoclassical tearing mode (NTM), energetic ion, tokamak

\section{Introduction}

The neoclassical tearing mode (NTM) is an important magnetohydrodynamics (MHD) instability, the presence of which can significantly limit the performance of high- $\beta$ plasma ( $\beta$ is a ratio of plasma thermal pressure to the magnetic pressure which is an important parameter to measure the confinement efficiency of a fusion device) [1]. The NTM is linearly stable, and is nonlinearly destabilized by a perturbed bootstrap current from the magnetic island, and the local flatting of current and pressure profile is the main difference from the classic tearing mode [2-3]. Both experiments and theories indicate that the width of a neoclassical magnetic island is proportional to the plasma poloidal $\beta_{p}$, and the scale length of safety factor gradient $L_{q}$, but inversely to the scale length of pressure gradient $L_{p}$ [4-6]. Based on understanding of NTM stability, active control has already made large progress and been broadly discussed in several devices such as DIII-D [7-8], ASDEX-Upgrade [9-10], JET [11], and JT-60U [12] etc.

Up to the present, it is still effective to use neutral beam injection (NBI) to obtain a high- $\beta$ discharge with flexible control of pressure 
profiles [13-14]. In this scenario, energetic ions are unavoidably brought into bulk plasmas. It was first observed in ASDEX-Upgrade that the neoclassical tearing mode interacted with energetic ions [15-17]. It was also reported that the trapped energetic ions resonate with a kink mode (fishbone [18] or off-axis fishbone [19-20]) leading to the frequency chirping-down MHD perturbations due to a wave-particle resonance [21-23]. It was then found that resonance can occur between circulating energetic ions and the kink modes [24-25]. On the other hand, energetic electrons have similar effects on kink modes due to drift-reversal for the barely trapped electrons or due to the negative magnetic shear for the passing particles [26-29]. Moreover, energetic electrons in the trapped-passing boundary are recently found to interact with a tearing mode leading to chirping-down of magnetic island rotation frequency in the lower collision region [30-32]. Furthermore, there are theories predicting that the energetic ions bring in kinetic effects on tearing modes (TMs) and NTMs [33-35]. However, experimental study is rare concerning the NTM dynamics influenced by energetic ions in tokamaks. This is probably due to the fact that the interaction only occurs near the island and is easily concealed by other strong interaction such as that between energetic ions and global kink modes (e.g. fishbone).

We describe the experimental scenario of NTM excitation in Section 2, show the effects of energetic ions on MHD modes in Section 3, and 
analyze the dynamics of NTM in Section 4. A summary will be presented in Section 5.

\section{Experimental scenario of NTM excitation}

The operation scenario in this study is as follows: the plasma current is $500 \mathrm{kA}$, the central line-average density is $\bar{n}_{e} \approx 2 \times 10^{19} \mathrm{~m}^{-3}$ by feedback control before entering $\mathrm{H}$-mode and ramps up to $\bar{n}_{e} \approx 3 \sim 4 \times 10^{19} \mathrm{~m}^{-3}$ during $\mathrm{H}$-mode phase (the electron temperature is near $T_{e} \approx 2 \mathrm{keV}$ in the core). Beside Ohmic heating the only auxiliary heating is the NBI system. The EAST-NBI has two beam lines locating at Ports A and F which are toroidally separated by 112.5 degree [36]. However, only the beam line at Port A has been used for the 2014 Fiscal Year campaign. This beam line has two ion sources, both of which are in the co-current direction. But, they have a separation of 8.7 degree in the toroidal direction [36-37]. In experiments, the operators usually start one ion source with a time delay (It is usually the less tangential one from the consideration of current drive and plasma stability.) after launching the first (more tangential). Interesting MHD activities were observed when the less tangential one was launched.

The MHD instabilities are summarized in the $\beta-l_{i}$ diagram $\left(l_{i}\right.$ is the plasma internal inductance which reflects the peaking degree of plasma current profile) as shown in figure 1 . Before the vertical lines in figure 1(a) and 1(b), the kink-like MHD oscillations are present and the 
internal inductance decrease with the plasma beta, even the torodial magnetic field, the plasma currents, and the plasma shaping parameters are controlled by setting the plasma control system (PCS). However, as the NBI (the less tangential beam line) is launched the plasma $\beta$ easily increase above unity for both the poloidal beta $\beta_{p}$ in figure 1(a) and the normalized beta $\beta_{N}\left(\beta_{N}=\beta[\%] /\left(I_{p}[M A] /(a[m] B[T])\right), \beta\right.$ is the ratio of the plasma pressure to the toroidal magnetic pressure, $I_{p}$ is the plasma current, $a$ is the minor radius and $B$ is the toroidal magnetic field.) in figure 1(b). The internal inductance $l_{i}$ no longer decreases with respect to the increasing $\beta_{p}\left(\beta_{p}\right.$ is the ratio of the plasma pressure to the poloidal magnetic pressure) and $\beta_{N}$. In particular, there are inflection points at $\beta_{p} \approx 1.1$ and $\beta_{N} \approx 1.1$. After these points are reached, it is observed that energetic ions interact with MHD modes including the kink modes and neoclassical tearing modes (the mode relevant to energetic particles is labeled as an EP-mode which is not the well-known energetic particle driven mode). Besides, strong sawtooth-like (ST-like) crashes are usually present. In figure 1(a), a slope line $\beta_{N}=2 l_{i}$ is particularly marked which basically passes through the statistical points indicating EP-modes and ST-like crashes at $\beta_{N}>1.5$. This relation is within the empirical scaling $\beta_{N} \leq 4 l_{i}$ for high- $\beta$ discharges [38].

A typical shot (49030) is shown in figure 2 which basically shows all characteristics of MHD instabilities indicated above. With the launching 
of less tangential beam line, the radiation of soft $\mathrm{x}$-ray (SX) in the core obviously increases in (b) which is accompanied by the decrement of plasma internal inductance $l_{i}$ in (a). This is easily understood because the plasma goes into the $\mathrm{H}$-mode phase as indicated by the sudden drop of $\mathrm{Da}$ radiation in (e). Before the first dash line at about $\mathrm{t}=5.2 \mathrm{~s}$, the normalized beta $\left(\beta_{N}\right)$ keep increasing towards the maximum $\beta_{N}=1.5$ in (d) while several regular sawtooth cycles are observed in (b) and there are no remarkable MHD perturbation on Mirnov signals in (c). After $t=5.2 \mathrm{~s}$, the $\beta_{N}$ decreases with $l_{i}$ while the regular sawtooth crash vanishes. Instead, two bursts of kink-like MHD oscillations appear. The burst is due to kinetic effects of particles either related to energetic ions or heavy impurities which have not been understood. Besides, three big edge localized modes (ELMs) trigger small crashes on the SX signals as indicated by the three dotted lines. Finally, a suddenly strong ST-like crash triggers strong MHD oscillations in (d) (This is the NTM which will be analyzed in the following sections).

This ST-like crash is not triggered by the ELM, which can be clarified in figure 3. Obviously, the ELM leads to the electron temperature decreasing at edge $(r / a \approx 0.7)$ indicated by the electron cyclotron emission (ECE) signals while the SX radiation diminishes a little in the core $(r / a \approx 0)$. However, these occurred before the sudden ST-like crash. Actually, there are precursor MHD modes which are observed on Mirnov 
signals, SX and ECE signals in the core. The ECE signals on available channels in plasma are shown in figure 4 where the precursor modes are clear in the channel $r / a \approx 0.4$ in (a). The fluctuations are weak on outer channels in (b) and (c). However, it can be recognized that the phase of oscillations are reversed in the channel $r / a \approx 0.4$ to $r / a \approx 0.52$ before the crash in (a). The contour plot of temperature fluctuation in (d) shows an inversion radius located between $r / a \approx 0.45$ and $r / a \approx 0.52$ which is estimated about $r_{i n v} / a \approx 0.47$ as indicated by the dash line. After the crash, a heat pulse is clearly propagating outwards from the inversion radius in (c) and (d). There is a local hot spot at the crash in (d) corresponding to the two channels of $r / a \approx 0.58$ and $r / a \approx 0.64$ in (b) which is actually due to a kinetic compression. This kind of phenomenon has been analyzed in previous paper [39], and will not be repeated here.

With above observations, it can be inferred that the center safety factor $q_{0}$ is below unity, and the $\mathrm{q}=1$ rational surface is at the phase-reversal surface $r_{i n v} / a \approx 0.47$ before the ST-like crash while the plasma current profile is broadened as indicated by the decreasing $l_{i}$. The evolution from the regular sawtooth cycles to the kink-like bursts indicates that the $q_{0}$ evolving towards $q_{0} \approx 1$, because the kink-like modes are easily destabilized and eventually trigger a strong ST-like crash for a flat q-profile with $q_{0} \rightarrow 1$ in present $\beta_{N}$ [40-42]. These kink-like modes are usually comprised of several harmonics $(\mathrm{m} / \mathrm{n}=1 / 1,2 / 2,3 / 3$, $\mathrm{m}$ and $\mathrm{n}$ is 
the mode number). Usually, the definition of nonresonant kink-like mode is used when the center safety factor moves to unity from above, because there is no $\mathrm{q}=1$ rational surface in the plasma [42-44]. In contrast, the center safety factor moves to unity from below in present experiments. We do not want to bring in a new name, and will use the 'kink-like' for modes with a flat central q-profile with $q_{0} \approx 1$. The direct measurement of center q is still a challenge in tokamaks and is absent for these shots.

The dynamics of MHD modes around the ST-like crash is indicated in figure 5. Before the ST-like crash, the frequency of precursor mode is about $7 \mathrm{kHz}$ as indicated in (b). The mode-induced fluctuations are phase-locked in the core SX and Mirnov signals as shown in (a) where the frequency spectrum is calculated by the Mrinov signals. At the crash there are obviously two harmonics besides the fundamental mode in the SX chord near the core in (b) and (c). In the more outer chord, the two harmonics are hard to differentiate and become connected together in the frequency spectrum as shown in (d). However, the Mirnov signals in (a) do not show the two harmonics indicating that the harmonic modes are located in the inner region. After the ST-like crash, the MHD oscillations diminish and even disappear on the outer chords of SX in (c) and (d) while perturbations survive in the core SX signals with the frequency of about $8.5 \mathrm{kHz}$ in (b). The Mirnov signals also show MHD oscillations in (a) where the frequency is lower than that in (b). It is also obvious that the 
raw signals show the different periods of perturbations on SX and Mirnov as indicated by two vertical dotted lines in (a). Figure 6 shows a comparison of MHD oscillations before (a) and after (b) the ST-crash on two chords. Raw signals obviously indicate the phase inversion at the vertical solid lines in (a). However, there are also two in-phase oscillations as marked by two vertical dash lines which are probably due to the existence of harmonics. In contrast, the fluctuations after the ST-like crash are basically in-phase with a shift in (b). The phase shift is caused by the different frequencies of those two signals as can be seen in figure 5 (b) and (d). Although there are also several oscillations of phase-inversion which we have not fully understood (One probable reason is indicated at the end of Sec.3), the compassion in figure (6) clearly shows that the $\mathrm{q}=1$ rational surface is moving inward during the ST-like crash and the MHD structure become kink-like after that crash. This again strengthens the evidence that the center safety factor is near unity around the ST-like crash.

The different frequencies after the ST-like crash imply the different modes in core (about $8.5 \mathrm{kHz}$ ) and at edge (about $5.5 \mathrm{kHz}$ ) which is proved by analyzing the multi-channel ECE signals in figure 7 . We extract MHD perturbations at $\mathrm{f}=5.5 \mathrm{kHz}$ and plot the contour of temperature fluctuations in (a). It is observed that there is a phase-inversion surface at $r / a \approx 0.77$ indicated by a horizontal solid line. 
Near that, there are obvious magnetic-island-induced fluctuations. However, the perturbed region in the inner is much broader than the outer perturbed region. This is actually due to the fact that this magnetic island is driven by a branch perturbation of the inner kink-like mode. It can be seen in figure $7(\mathrm{~b})$ where fluctuating signals covering the spectrum of $7 \sim 8.5 \mathrm{kHz}$ are extracted. The reason to choose this frequency band is that the previous analysis indicates the frequency decreases from the core to edge as seen in the figure 5 (b) and (d). The kink-like perturbation is clearly located within the region of $r / a=0.5$ while there is another perturbed branch in $r / a=0.7$ indicated by a solid line in figure 7(b). The profiles of perturbation amplitudes are shown in figure 7(c). The value of core perturbation within $r / a=0.5$ is larger than that around $r / a \approx 0.77$. The latter is obviously more localized $(\Delta r / a \approx 0.15)$ around a rational surface demonstrating a characteristic of magnetic island. Actually, the MHD mode is a $\mathrm{m} / \mathrm{n}=2 / 1$ mode by analyzing spatially distributed Mirnov coils which rotates in the direction of ion diamagnetic drift. The excitation is due to the residue perturbation near the $\mathrm{q}=2$ rational surface which is driven by the $m / n=1 / 1$ kink-like mode in the core. By the way, the excitation of NTM by mode coupling has been observed in EAST previously [45].

\section{Effects of energetic ions on MHD modes}

The interaction of energetic ions with MHD instabilities is shown in 
figure 8. Before the ST-like crash as indicated by the vertical dash line L1, the effect of energetic ions leads to the frequency chirping-down evolution of SX signals in (a) and ssNPA (solid state Neutral Particle Analyzer) signals in (b). It does not result in any measurable magnetic perturbation on Mirnov signals in (c). ssNPA measures the lost fast neutral particles and also reflects the presence of energetic ions in plasma to some extend [46]. The frequency spectrum of ssNPA fluctuations shows continuing chirping-down evolutions round $\mathrm{f}=21 \mathrm{kHz}$ which is the precession frequency of $\sim 22 \mathrm{keV}$ energetic ions in current experimental condition. On the other hand, the frequency spectrum of SX fluctuations shows the similar behavior except the phase during the ELM burst in (a). During the ELM burst indicated by the Da signals, the frequency of ssNPA signal is uplifted while the corresponding change is absent on SX signals. The chirping-down evolution of SX spectrum comes from the interaction of confined energetic ions with the precursor modes while ssNPA actually measures the escaped energetic neutral particles. After the ST-like crash, the chirping-down phenomenon on the SX frequency spectrum still exists while that is nearly absent on ssNPA spectrum except two sparse bursts. This illustrates that the energetic ions are reduced after ST-like crash due to redistributions. In particular, the NTM appears as teeth-like oscillations on the spectrum of Mirnov signals (periodic frequency humps) in (c), which does not lead to extra loss of energetic 
ions. This is probably due to the fact that the NTM exists in the outer region $(r / a \approx 0.77)$ away from the major confinement region of energetic ions.

This can be further illustrated in comparing frequency spectra of SX signals on different chords from the plasma core to edge in figure 9. Figure 9 (a) shows two frequency bands at $\mathrm{f}=8.5 \mathrm{kHz}$ and $\mathrm{f}=17 \mathrm{kHz}$. After $\mathrm{t}=5.44 \mathrm{~s}$, the frequency spectrum at the lower frequency band is smooth which does not show interaction of energetic ions with the mode. Actually it is a kink-like mode which still exists after the ST-like crash. However, the frequency $(\mathrm{f}=17 \mathrm{kHz})$ of its secondary harmonic is near the bottom of the chirping-down frequency dragged down by energetic ion precession. The effect of energetic ions gets stronger on more outer chord $(r / a \approx 0.36)$ while the amplitude of fundamental mode decays in figure 9(b). Further outward at $r / a \approx 0.39$ in (c), the interaction becomes very strong showing obvious chirping-down evolutions round $\mathrm{f}=17 \mathrm{kHz}$. The frequency evolution of the fundamental mode $(\mathrm{f}=8.5 \mathrm{kHz})$ is also frequently interrupted. The interaction of energetic ions with the mode at $\mathrm{f}=17 \mathrm{kHz}$ is clearly observed on the chord of $r / a \approx 0.42$ in (d) while it becomes weak on most outer chords in figures 9 (e) and (f) where the interaction with fundamental mode is hardly observed. These observations demonstrate that the energetic ions are located at $r / a \approx 0.39 \sim 0.42$ induced by NBI heating. The activity that energetic ions 
frequently interrupts the frequency evolution of fundamental mode $(\mathrm{f}=8.5 \mathrm{kHz})$ is clearly shown on ECE signals in figures 10 (a) and (b). Figure 10(a) shows the ECE fluctuations and their frequency spectrum in the most inner available channel $(r / a \approx 0.4)$. The modulation of frequency is very clear which is also remarkable in the more outward channel $(r / a \approx 0.45)$ in figure $10(\mathrm{~b})$. However, the harmonic $(\mathrm{f}=17 \mathrm{kHz})$ of $\mathrm{SX}$ signals is not observed in the ECE frequency spectrum. The main reason is that the ECE is a local measurement while the SX is a chord-integral one along the sightline. The secondary harmonic is probably located more inward. The figure 10(c) shows the ECE raw signals and their frequency spectrum at $r / a \approx 0.7$ which are different from those above in (a) and (b). It is obvious that the oscillations in (c) start from the ST-like crash with a frequency band round $\mathrm{f}=5.5 \mathrm{kHz}$ (the $\mathrm{m} / \mathrm{n}=2 / 1 \mathrm{NTM}$ ). The evolution of frequency demonstrates some humps which will be analyzed in Sec. 4.

It has been indicated in figure 7 that the NTM is located round $r / a \approx 0.77$ driven by the residue magnetic perturbation after the ST-like crash. It is also illustrated that the kink-like mode is located inside of $r / a=0.5$. Besides, the figure 10 particularly indicates that both the kink-like mode inside and the NTM outside are influenced by the kinetic effects of energetic ions. The subsequent evolution of the kink-mode and NTM is illustrated in figure 11. The figure 11(a) is the contour plot of ECE raw signals where the rational surface is indicated by the horizontal 
line. The vertical dash lines indicate the perturbations induced by the NTM which can be clearly observed in figure 11(b). The radial width of perturbations is about $\Delta r / a \approx 0.15$ which is nearly equal to that in figure 7. Besides, it seems that there are other weak perturbations as indicated by the horizontal dash line. The radial location is occupied by the kink-like mode as indicated in figure 11(c). In contrast to that in figure 7, it is obvious that the NTM interacting with energetic ions provides a perturbation to the kink-like mode in this phase. This may explain why both in-phase and phase-reversal oscillations coexist after the ST-like crash in figure 6.

\section{Analysis of the NTM dynamics}

The interaction of energetic ions with the NTM does not lead to the usual frequency chirping down on the time frequency spectrum of fluctuating signals. One of reasons is that the location of the NTM is away from the major confinement region of energetic ions so that the direct resonant interaction via wave-particle can not occur. On the other hand, even in the major confinement region of energetic ions, it was observed that the frequency chirping-down only occurred around the secondary harmonic of kink-like mode while the fundamental mode was weakly modulated (The frequency spectrum does not show the chirping-down evolutions).

The frequency (about $\mathrm{f}=17 \mathrm{kHz}$ ) is not equal to the value of precession frequency of energetic ions in current energy range $(\mathrm{f}=21 \mathrm{kHz})$ which can 
be observed on spectrum of SX and ssNPA signals before the ST-like crash. It should be noted that the ST-like crash redistributes energetic ions modifying the precession frequency, and the evolution of the q-profile after the ST-like crash may also change the frequency of the mode a little. Nevertheless, the characteristic of frequency evolution in figure 9 implies that the kink-like mode belongs to MHD mode with frequency dominated by the bulk plasma, different from the energetic-ion-driven modes whose frequencies are determined by the energetic ions [47]. This is probably due to the fact that the population of trapped energetic ions is small in current NBI heating, easily thermalized by collisions with the bulk plasma reducing the resistivity. This is illustrated by the continual decrement of internal inductance. Of course, the effect of the fast ion current drive is not neglected. As noted in previous papers [31-32], the collisionality is a very important parameter for investigating the interaction between energetic particles with MHD modes. In the present scenario, a higher collisionality is anticipated because the parameters (lower temperature and higher density) of the bulk plasma are not enough high and the plasma beta $(\beta)$ is still marginal to trigger these MHD instabilities. From this aspect, the modes mainly reflect the MHD behaviors of the bulk plasma, with frequencies weakly modulated by energetic ions. In this paper, the interaction of kink-like fundamental mode with the energetic ions will not be further analyzed. Instead, we 
focus on the NTM dynamics.

As indicated previously, the rational surface (round $r_{s} / a \approx 0.77$ ) of the NTM is far away from the major confinement region (round $r / a \approx 0.39 \sim 0.42$ ) of energetic ions. The width of banana orbits is about $\Delta r / a \approx 0.3$ for the trapped energetic ions in the energy range of $22 \mathrm{keV}$ which will be shown by the calculation of energetic ion orbits. Since energetic ions are not monoenergetic, their orbits are not fully overlapped. Thus, the effect of energetic ions on the NTM is not localized at the rational surface of the mode. Figure 12 (a) shows the spectrum and the raw ECE signal at $r / a \approx 0.7$ near the rational surface where the NTM-induced temperature fluctuations are demonstrated by irregular humps on the frequency spectrum (there are also three chirping processes, the intensity of which are very weak in comparison to those of humps). Figure 12 (b) show the NTM mode measured by Mrinov signals where modifications of magnetic signals on both the frequency and the width of island denoted by the square root value of perturbed amplitude are very regular as indicated by the dash lines (the frequency increase from the baseline $\mathrm{f}=5.5 \mathrm{kHz}$ to $\mathrm{f}=7.5 \mathrm{kHz}$ ). Figure 12 (c) shows the NTM-induced fluctuations of the electron temperature at $r / a \approx 0.7$ where the envelope of oscillating period basically agrees with the magnetic one. The difference lies at $\mathrm{t}=5.4315 \mathrm{~s}-5.4325 \mathrm{~s}$ when the period of temperature fluctuation merges two magnetic ones. Errors may be there due to 
indistinguishable peaks induced by the fast growing perturbations while the essence is attributed to the local change of energetic ions. However, the Mirnov coil measures magnetic perturbations without radial resolution, leading to less influence.

The banana orbits of energetic ions are analyzed by the orbit code 'ORBIT' which was developed in 1980's [48]. The enrichment by considering the effect of MHD perturbations on particle dynamics was also finished recently [49]. This code is applied to check the change of energetic ion orbits. The results are shown in figure 13 where the orbit without NTM perturbations is illustrated in (a). The banana orbit is closed (the staring and ending points repeat). However, once a magnetic island is included the orbit demonstrates a radial shift (i.e. excursion) as illustrated by the arrow in figure 13(b). The radial shift of energetic ion orbits would result in a neoclassical polarization current which is as follows [50]:

$$
j_{r}=d E_{r} / d t\left(\rho_{m} c^{2} / B_{\theta}^{2}\right)
$$

$\rho_{m}$ is the mass density. The time-varying radial electrical field has the following relationship with the bounce-averaging radial velocity when one considers the conservation of toroidal angular momentum [50]:

$$
V_{r} \approx \frac{m c^{2}}{e B_{\theta}^{2}} d E_{r} / d t
$$

With a specific shape of electric potential (the gradient of electric potential is the electrical field), it was demonstrated that the radial motion of energetic ions modifies not only the width of neoclassical island but 
also the rotation frequency [51-53]:

$$
\begin{gathered}
\frac{d}{d t}\left(\Omega-\Omega_{1}\right)=\frac{1}{w}\left(\frac{1-\Omega}{\sqrt{\Omega}} w^{3}+C_{2}(w, \Omega)-\left(\Omega-\Omega_{1}\right) \frac{d w}{d t}\right) \\
\frac{d w}{d t}=v\left(r_{s} \Delta^{\prime}+\frac{\beta_{p}}{2} \frac{a_{b s}-a_{G G J}}{2 r_{s} w}+\frac{C_{3}(\Omega)}{\left(2 r_{s} w\right)^{3}} \Omega\left(\Omega-\Omega_{1}\right)\right)
\end{gathered}
$$

The coupled equations (3) and (4) describe the mutual dependence of the frequency and the width of magnetic island. The $\Omega$ is the normalized value of the mode frequency to the thermal ion diamagnetic frequency $\Omega=\omega /\left(\omega_{s_{i}}\left(1+0.39 \eta_{i}\right)\right)$ where the $\eta_{i}$ is the ratio of the density scale length to the ion temperature scale length. The $\Omega_{1}$ is defined as $\Omega_{1}=\left(1+\eta_{i}\right) /\left(1+0.39 \eta_{i}\right)$ while the $v$ is related to the resistive diffusion time $v=\left(v_{c} \tau_{R}\right)^{-1}$. The time variable has been normalized by the frequency parameter $v_{c}$. The parameter $w$ describes the width $W$ of magnetic island through $w=W / 2 r_{s}$. The coefficient $C_{2}(w, \Omega)$ is a function of frequency and width in second term of right hand side (RHS) in Eq.(3) which indicates the direct action of energetic population on the rotation frequency. The coefficient $C_{3}(\Omega)$ in Eq.(4) reflects the influence of energetic ions on the width of magnetic island which is involved with the frequency. The other parameters in Eq.(4) have the usual definition: $\Delta$ the stability parameter of classical tearing mode, $r_{s}$ the radius of rational surface, $\beta_{p}$ is the poloidal beta, $a_{b s}$ the driving term of bootstrap and $a_{G G J}$ the Glasser-Green-Johnson stabilizing term due to toroidal effect. 
Numerical calculations based on Eqs. (3) and (4) have been done to compare evolutions of island width and frequency with experimental observations. In present experiments, the modification of frequency by energetic ions is weak as shown in figure 12(c). It is thus rational to assume the coefficient $C_{2}(w, \Omega)=0$ in Eq.(3). This means that the frequency is only influenced by the width of magnetic island round its initial value. However, we keep the third term of RHS in Eq.(4) where the modifying coefficient is supposed as $C_{3}(\Omega)=0.1 * \cos (\Omega t)$ due to the periodic interaction of energetic ions with the NTM. This includes two effects of energetic ions: the destabilization of ion polarization current (positive semi-period of $C_{3}$ ) and its threshold to the energetic ion pressure (The negative semi-period of $C_{3}$ means that the threshold is not surpassed.). The later comes from the fact that the energetic ions drifts from inside to outside of the rational surface, periodically modifying the pressure profile. The stability parameter of tearing mode is assumed $\Delta^{\prime} \approx-m=-2$ which means that the NTM is linearly stable. The rational surface is estimated at $r_{s}=0.77 a$ where $a$ is the minor radius of plasma. The ratio of scale lengths is assumed as $\eta_{i}=1.5$ and the poloidal beta is $\beta_{p}=1.2$. Since the NTM is triggered by the ST-like crash, we thus assume that the combining effect of the bootstrap driving term and the Glasser-Green-Johnson stabilizing term is destabilizing $a_{b s}-a_{G G J}=0.05$. With suppositions of $\tau_{R}=10^{3} \mathrm{~ms}, \quad v=10^{-3}$, and $\omega_{*_{i}}=2 \mathrm{kHz}$, the calculating 
results are scaled in the usual laboratory units (the time unite is millisecond and the frequency unit is kilohertz) which are illustrated in figure 14. It is obvious that the width of magnetic island oscillates around the value $\Delta r / a=0.15$, in synchronization with the rotation frequency varying around $\mathrm{f}=6 \mathrm{kHz}$. These agree with experimental observations in figures 11 and 12.

It is also necessary to indicate that the onset of the NTM mode needs the trigger of ST-like crash in these experiments. The above analysis only clarifies the important effect of energetic ions on the nonlinear evolution, not referring to the onset of NTM. However, the energetic ions polarization current may be significant to that when the rational surface of NTM is located in the confinement region of energetic ions. This will be further analyzed in comparative experiments.

\section{Summary}

The dynamics of the NTM has been analyzed in detail including its excitation which is triggered by the ST-like crash and the characteristic of nonlinear evolution which is modified by energetic ions. The interaction of energetic ions with the NTM is weaker than that with the kink modes. Analysis indicated that the kink modes locate at a more inner region where trapped energetic ions are confined. In contrast, the NTM exists at a more outer region which is influenced by the radial excursion of energetic ion orbits. Both the width of neoclassical island and the rotation 
frequency are modulated by periodic perturbations of energetic ion polarization current. No remarkable resonance is observed between energetic ions and the NTM as with the kink modes in current operating scenario. The interaction of energetic ions with the kink modes is also different from the conventional fishbone mode. The main difference is that our experiments here do not have a $\mathrm{q}=1$ rational surface in the plasma after the ST-like crash. Only the second harmonic of kink mode interacts with energetic ions leading to the chirping-down frequency evolution due to its frequency near the precession frequency of energetic ions. A further study is necessary to clarify the influence of operating scenario on core MHD instabilities and energetic particles.

\section{Acknowledgment}

This work is supported by the National Natural Science Foundation of

China under Grant No. 11405216, 11575249, 11175209, partially by the JSPS-NRF-NSFC A3 Foresight Program in the field of Plasma Physics (NSFC No 11261140328) and by the National Magnetic Confinement Fusion Energy Research Program under Grant No. 2015GB110005, 2015GB101000 .

\section{References}

[1] ITER Physics Basis, 2007 Nucl. Fusion 47 S128

[2] Hegna C, 1998 Phys. Plasmas 5, 1767

[3] Rutherford P, 1973 Phys. Fluids 16, 1903 
[4] Buttery R, Hender T, Howell D, La Haye R, Sauter O, Testa D, 2003 Nucl. Fusion 43, 69

[5] Zohm H, Gate D, Wilson H, Gantenbein G, Gruber O, Gunter S, Maraschek M, et al, 1997 Plasma Phys. Control. Fusion 39 B237

[6] Carrera R, Hazeltine, Kotschenreuther M, 1986 Phys. Fluids 29899

[7] La Haye R, Gunter S, Humpreys D, Lohr J, Luce T, Maraschek M, Petty C, Prater R, Scoville J, Strait E, 2002 Phys. Plasmas 92051

[8] La Haye R, 2006 Phys. Plasmas 13055501

[9] Zohm H, Gantenbein G, Gude A, Gunter S, Leuterer F, Maraschek M, Meskat J, Suttrop W, Yu Q, 2001 Phys. Plasmas 82009

[10] Yu Q, Zhang X, Gunter S, 2004 Phys. Plasmas 111960

[11] Sauter O, Westerhof E, Mayoral M, Alper B, Belo P, Buttery R, Gondhalekar A, Hellsten T et al, 2002 Phys. Rev. Lett. 88105001

[12] Isayama A, Kamada Y, Ide S, Hamamatsu K, Oikawa T, Suzuki T, Neyatani Y, Ozeki T, Ikeda Y, Kajiwara K, 2000 Plasma Phys. Control. Fusion 42 L37

[13] Isayama A, Kamada Y, Hayashi N, Suzuki T, Oikawa T, Fujita T, Fukuda T, Ide S, Takenaga H, Ushigusa K, Ozeki T, Ikeda Y, Umeda N, Yamada H, Isobe M, Narushima Y, Ikeda K, Sakakibara S, Yamazaki K, Nagasaki K, 2003 Nucl. Fusion 431272

[14] Isayama A, Kamada Y, Ozeki T, Ide S, Fujita T, Oikawa T, Suzuki T, Neyatani Y, Isei N, Hamamatsu K et al, 2001 Nucl. Fusion 41761 
[15] Sesnic S, Gunter S, Gude A, Maraschek M, 2000 Phys. Plasmas 7 935

[16] Gunter S, Gude A, Maraschek M, Sesnic S, Zohm H, 2001 Phys. Rev. Lett. 87275001

[17] Gunter S, Maraschek M, de Baar M, Howell D, Poli E, Strumberger E, Tichmann C, 2004 Nucl. Fusion 44524

[18] McGuire K, Goldston R, Bell M, Bitter M, Bol K, Brau K, Buchenauer D, Crowley T et al, 1983 Phys. Rev. Lett. 50891 [19] Okabayashi M, Matsunaga G, deGrassie J, Heidbrink W, In Y, Liu Y, Reimerdes H, Solomon W et al, 2011 Phys. Plasmas 18056112 [20] Heidbrink W, Austin M, Fisher R, Garcia-Munoz M, Matsunaga G, McKee G et al, 2011 Plasma Phys. Control. Fusion 53085028

[21] Chen L, White R, Rosenbluth M, 1984 Phys. Rev. Lett. 521122

[22] White R, Chen L, Romanelli F, Hay R, 1985 Phys. Fluids 28278

[23] Coppi B, and Porcelli F, 1986 Phys. Rev. Lett. 572272

[24] Wang S, 2001 Phys. Rev. Lett 865286

[25] Gobbin M, White R, Marrelli L, Martin P, 2008 Nucl. Fusion 48 075002

[26] Wong K, Chu M, Luce T, Petty C, Politzer P, Chen L, Harvey R, Austin M, Johnson L, La Haye, R, Snider, 2000 Phys. Rev. Lett. 85996

[27] Wang Z, Long Y, Wang A, Dong J, Wang L, Zonca F, 2007 Nucl. Fusion 471307 
[28] Chen W, Ding X, Liu Y, Yuan G et al, 2009 Nucl. Fusion 49075002

[29] Zonca F, Buratti P, Cardinali A, Chen L, Dong J, Long Y, Milovanov A, Romanelli F, Smeulders, Wang L et al, 2007 Nucl. Fusion 471588

[30] Chen L and White R, 1977 Phys. Rev. Lett. 39460

[31] Li E, Lin S, Shen B, Hu L, 2015 Nucl. Fusion 55063024

[32] Li E, Hu L, Lin S, Shen B, Liu Y, 2014 Nucl. Fuison 54042001

[33] Hegna C, Bhattacharjee A, 1989 Phys. Rev. Lett. 632056

[34] Cai H, Wang S, Xu Y, Cao J, Li D, 2011 Phys .Rev. Lett. 106075002

[35] Cai H and Fu G, 2012 Phys. Plasmas 19072506

[36] Hu C, 2012 Plasma Sci. Technol. 14871

[37] Lee M, Wu B, Wu X, Li H, 2014 J. Fusion Energ. 33549

[38] Strait E, Taylor T, Turnbull A, Ferron J, Lao L, Rice B, Sauter O, Thompson S and Wroblewski D, 1995 Phys. Rev. Lett. 742483

[39] Li E, Hu L, and Chen K, 2014 Phys. Plasmas 21012501

[40] Xu L, Hu L, Chen K, Li E et al, 2012 Phys. Plasmas 19122504

[41] Wang Z, Wai L, and Wang X, 2012 Phys. Plasmas 19062108

[42] Li E, Igochine V, Dumbrajs O, Xu L, Chen K, Shi T, Hu L, 2014 Plasma Phys. Control. Fusion 56125016

[43] Breslau J, Chance M, Chen J, Fu G, Gerhardt S, Gorelenkov N, Jardin S, and Manickam J, 2011 Nucl. Fusion 51063027

[44] Chapman I, Hua M, Pinches S, Akers R, Field A, Graves J, Hastie R, Michael C, 2010 Nucl. Fusion 50045007 
[45] Shi T, Wan B et al, 2013 Plasma Phys. Control. Fusion 55055007

[46] Zhu Y, Zhang J et al, 2014 Rev. Sci. Instrum. 85 11E107

[47] Heidbrink W, Gorelenkov, Murakami M, 2002 Nucl. Fusion 42972

[48] White R and Chance M, 1984 Phys. Fluids 272455

[49] White R, 2011 Plasma Phys. Control. Fusion 53085018

[50] Hinton F and Robertson J, 1984 Phys. Fluids 271243

[51] Marchenko V and Lutsenko V, 2001 Phys. Plasmas 84834

[52] Smolyakov A, Hirose A, Lazzaro E, Re G, Callen J, 1995 Phys. Plasmas 21581

[53] Wilson H, Connor J, Hastie R, Hegna C, 1996 Phys. Plasmas 3248

\section{Figure captions}

Figure 1. The statistical results of MHD modes in the 2014 Fiscal Year's campaign of EAST in the diagram of the plasma internal inductance $l_{i}$ and plasma normalized beta $\beta_{N}$ (a), the product $l_{i} * q_{95} \quad\left(q_{95}\right.$ is the safety factor at $95 \%$ poloidal flux) and plasma poloidal beta $\beta_{p}(\mathrm{~b})$. The vertical lines mark an inflection point, before which the $l_{i}$ and $l_{i} * q_{95}$ decrease with the $\beta_{N}$ and $\beta_{p}$ in (a) and (b), respectively. Above $\beta_{N}>1.4$, a relationship $\beta_{N}=2 l_{i}$ is labeled across the statistical points in (a) where MHD modes related to energetic particles (EP-mode) and sawtooth-like (ST-like) crashes exist.

Figure 2. A typical discharge (\#49030): (a) the plasma internal inductance, (b) the soft X-ray (SX) radiation on the core chord with the NBI of less 
tangential angel marked, (c) the Mirnov signals, (d) the normalized beta $\beta_{N}$ and (e) the Da signals. The two vertical dash lines indicate two different types of crashes. The first one is the regular sawtooth crash while the second indicates the strong ST-like crash triggering the NTM. The three dotted lines mark three larger edge localized modes (ELMs) which lead to weak decrease of SX radiations in (b). The vertical solid line indicates the H-L back transition.

Figure 3. The details of the ST-like crash. The strong crash is after the ELM which is resulted by the precursor modes. The precursors are also measured by the Mirnov coils which are differentiated from the following NTM perturbations. The ELM leads to a little decrease of SX in the core and obvious drop of electron cyclotron emission (ECE) signals on the outer channel $r / a=0.7$.

Figure 4. The MHD perturbations around the ST-like crash on available ECE channels: (a) at $r / a=0.4, \quad r / a=0.45$ and $r / a=0.51$ where the phase-reversal oscillations are observed before the ST-like crash between $r / a=0.4$ and $r / a=0.51$, (b) at $r / a=0.58, \quad r / a=0.64$ and $r / a=0.68$ where there are strong prompt radiation at the crash, (c) at $r / a=0.7$, $r / a=0.79, r / a=0.84, r / a=0.9$, and $r / a=0.94$ where the diffusion of a heat pulse is indicated by the slope arrow. The contour plot is shown in (d) where the inversion surface $\left(r_{i n v} / a \approx 0.47\right.$ ) of oscillating phases is labeled by the horizontal dash line. The color bar is ignored, but colors from blue 
to red indicate the perturbed amplitude from negative to positive values. Figure 5. The characteristics of MHD modes around the ST-like crash: the time frequency spectrum of Mirnov signals where the raw signals of Mirnov and SX are superimposed (a), the frequency spectra with SX raw signals on the chord $r / a \approx 0(\mathrm{~b}), r / a \approx 0.2(\mathrm{c})$ and $r / a \approx 0.51(\mathrm{~d})$. The vertical dash lines indicate the phase-locked relationship on SX and Mirnov before the ST-like crash while this is broken after the crash as illustrated by the two dotted lines in (a). There are harmonics of $m / n=1 / 1$, $2 / 2,3 / 3$ around the crash in (b), (c), (d). The frequency of fundamental mode in (b) increases from about $7 \mathrm{kHz}$ to $8.5 \mathrm{kHz}$ while that weakly increases to about $7 \mathrm{kHz}$ in (d). After the ST-like crash, the MHD oscillations are remarkably stronger in (a) whereas the core fluctuations become weak or even unobservable in (c) and (d). Contours use the same color bar.

Figure 6. The comparison between oscillation phases (at $r / a \approx 0$ and at $r / a \approx 0.51$ ) before (a) and after (b) the ST-like crash. The vertical solid lines indicate the fluctuations of phase reversal while the vertical dash lines mark the in-phase (or with a fixed shift) oscillations. Existence of two in-phase oscillations is due to harmonics in (a). The oscillations at both two locations basically keep a fixed phase shift just after the crash in (b). However, the following oscillations evolve with in-phase and reversed phase due to the mode coupling between the core and edge. 
Figure 7. The spatial structure of the MHD modes after the ST-like crash: (a) the demonstration of the NTM at $r_{s} / a \approx 0.77$ as indicated by the horizontal solid line which is obtained at the preferred frequency of $\mathrm{f}=5.5 \mathrm{kHz}$ by Fast Fourier Transformation (FFT) of multi-channel ECE fluctuations, (b) the existence of a kink mode at $r / a=0.5$ with a branch perturbation at $r / a=0.7$ marked by a horizontal solid line at the frequency band of $\mathrm{f}=7 \sim 8.5 \mathrm{kHz}$, and (c) the profiles of perturbed amplitudes corresponding to the NTM (symbols like solid circles) and the kink mode (symbols like squares). The NTM is driven by the branch perturbation of the kink mode. Contours use the same color bar.

Figure 8. The influence of energetic ions on the MHD dynamics: (a) the raw SX signal at $r / a \approx 0.22$ and its frequency spectrum, (b) the ssNPA signal and its frequency spectrum with the core SX signal superimposed, (c) the Mirnov signal and its frequency spectrum with the core SX signal superimposed. The vertical dash line L1 marks the ST-like crash which triggers the NTM, not the ELM indicated by the burst of Da signals in (a) and (c). The vertical dash line L2 states that the NTM is stabilized in (c) while the kink mode and its harmonic is alive in (a). The chirping down evolution on spectrum indicate the existence of energetic ions in (b) and the interaction with the secondary harmonic of kink mode in (a) which is near the precession frequency of energetic ions in the energy of $22 \mathrm{keV}$. The evolution of the NTM frequency spectrum exhibits regular teeth-like 
modulations implying the effect of energetic ions. Contours use the same color bar.

Figure 9. The comparison of the frequency spectra of SX signals at: (a) $r / a \approx 0.14$, (b) $r / a \approx 0.36$, (c) $r / a \approx 0.39$, (d) $r / a \approx 0.42$, (e) $r / a \approx 0.45$ and (f) $r / a \approx 0.51$. The frequency chirping-down evolutions are obvious in (c) and (d) round the frequency of secondary harmonic of kink mode. For the inner region in (a) and (b), the harmonic frequency seems modulated a little without the chirping-down evolutions while the fundamental mode is less influenced with a smooth evolution at $\mathrm{f}=8.5 \mathrm{kHz}$. For the outer region in (d) and (e), the chirping down frequency also seems weak and sparse with time. Contours use the same color bar.

Figure 10. The comparison of ECE fluctuations and their frequency spectra at: (a) $r / a \approx 0.4$, (b) $r / a \approx 0.45$, and (c) $r / a \approx 0.7$. The spectrums in (a) and (b) show the frequency $\mathrm{f}=8.5 \mathrm{kHz}$ of kink mode which is frequently interrupted indicating the effect of energetic ions. The activity of NTM is shown in (c) the frequency of which is about $\mathrm{f}=5.5 \mathrm{kHz}$ with some humps. The humps on the frequency evolution imply the interaction of energetic ions with the NTM. Contours use the same color bar.

Figure 11. The spatial structure of ECE fluctuations at subsequent phase of the evolution in figure 7: (a) the contour plot of raw signals, (b) the NTM perturbations extracted by FFT at $\mathrm{f}=5.5 \mathrm{kHz}$, and (c) the kink mode at $\mathrm{f}=7-8.5 \mathrm{kHz}$ within $r / a=0.5$. The vertical dash lines indicate the 
phase-reversal perturbations near the rational surface as indicated by the horizontal solid lines in (a) and (b). The horizontal dash line in (b) marks weak perturbations in the radial region of kink mode. Contours use the same color bar.

Figure 12. The frequency spectrum of ECE fluctuations with raw signals superimposed in (a), the details of NTM dynamics measured by Mirnov signals in (b), and the electron temperature fluctuations and the envelope evolution at $r / a \approx 0.7$ in (c). The periodic evolutions of rotation frequency and island width (denoted by the square root of the perturbed amplitude) in (b) agree with the varying envelope of NTM-induced electron temperature fluctuations in (c), demonstrating effects of energetic ions. Contours use the same color bar.

Figure 13. The calculated orbits of trapped energetic ions at the energy of $22 \mathrm{keV}$ without (a) and with (b) the $\mathrm{m} / \mathrm{n}=2 / 1$ tearing mode. Calculations are done by using the ORBIT code where a broadened q-profile has been polynomial-fit with experimental points $\left(q_{95}=4.8, q(r / a=0.77)=2\right.$, and assuming $q_{0}=1.01$ due to the absence of $\mathrm{q}=1$ in plasmas after the ST-like crash). It uses the usual odd-function displacement of the tearing mode with the perturbed magnetic field limited at edge by the measured value (0.22Gauss). In comparison to that in (a), the orbit of energetic ions has a radial shift (excursion) due to the existence of tearing mode.

Figure 14. The calculated width of magnetic island (a) and the rotation 
frequency (b) based on the coupled equations eqs. (3) and (4). The effect of energetic ions is assumed to modulate the width of magnetic island with considering the periodic excursion of orbit (supposing $\left.C_{3}(\Omega)=0.1^{*} \cos (\Omega t)\right)$. The direct action of energetic ions on the frequency is neglected $\left(C_{2}(w, \Omega)=0\right)$ according to experimental observations while the frequency modulation is self-consistently done by varying island width. The other parameters are set as: $\Delta^{\prime} \approx-m=-2, r_{s}=0.77 a, \eta_{i}=1.5$, $\beta_{p}=1.2, \quad a_{b s}-a_{G G J}=0.05, \tau_{R}=10^{3} \mathrm{~ms}, \quad v=10^{-3}, \omega_{* i}=2 \mathrm{kHz}$. With these parameters, the oscillation period agrees with the experimental measurement in figure 12(c). The evolution of frequency is basically consistent with the humps of frequency in figure 12 (c) while the width of magnetic island oscillates around the $\Delta r / a=0.15$ agreeing to that in figure 7(a).

\section{Figures}



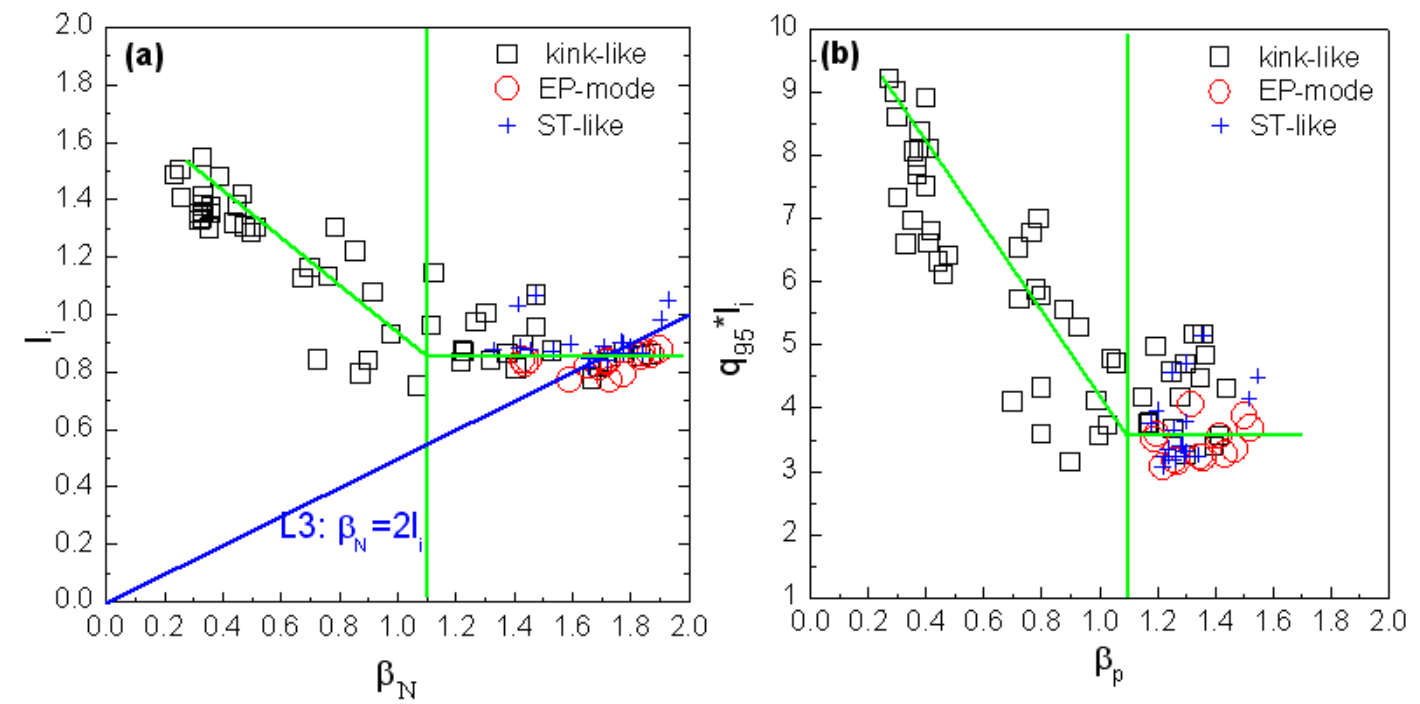

Figure 1 
shot: 49030

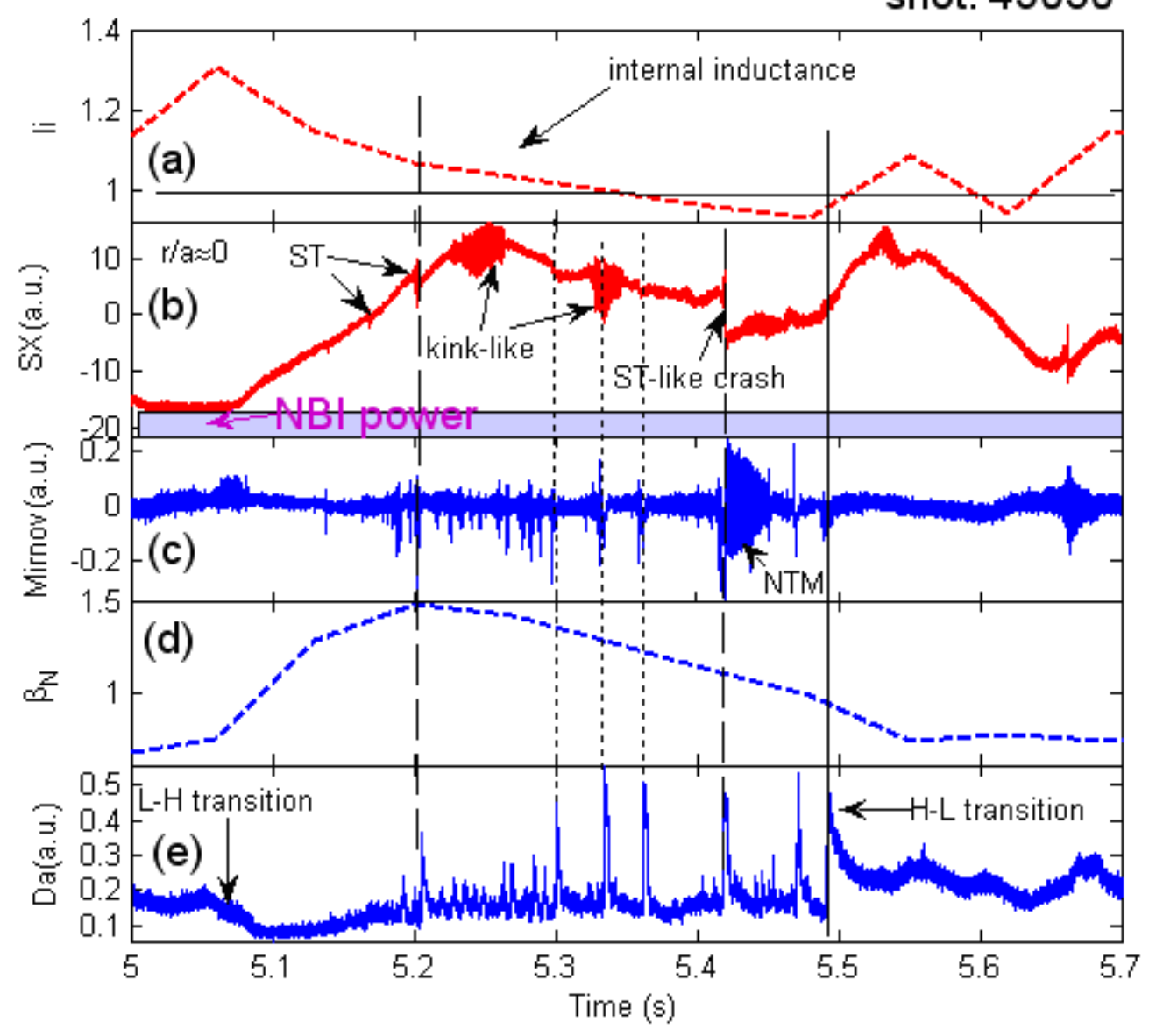

Figure 2 


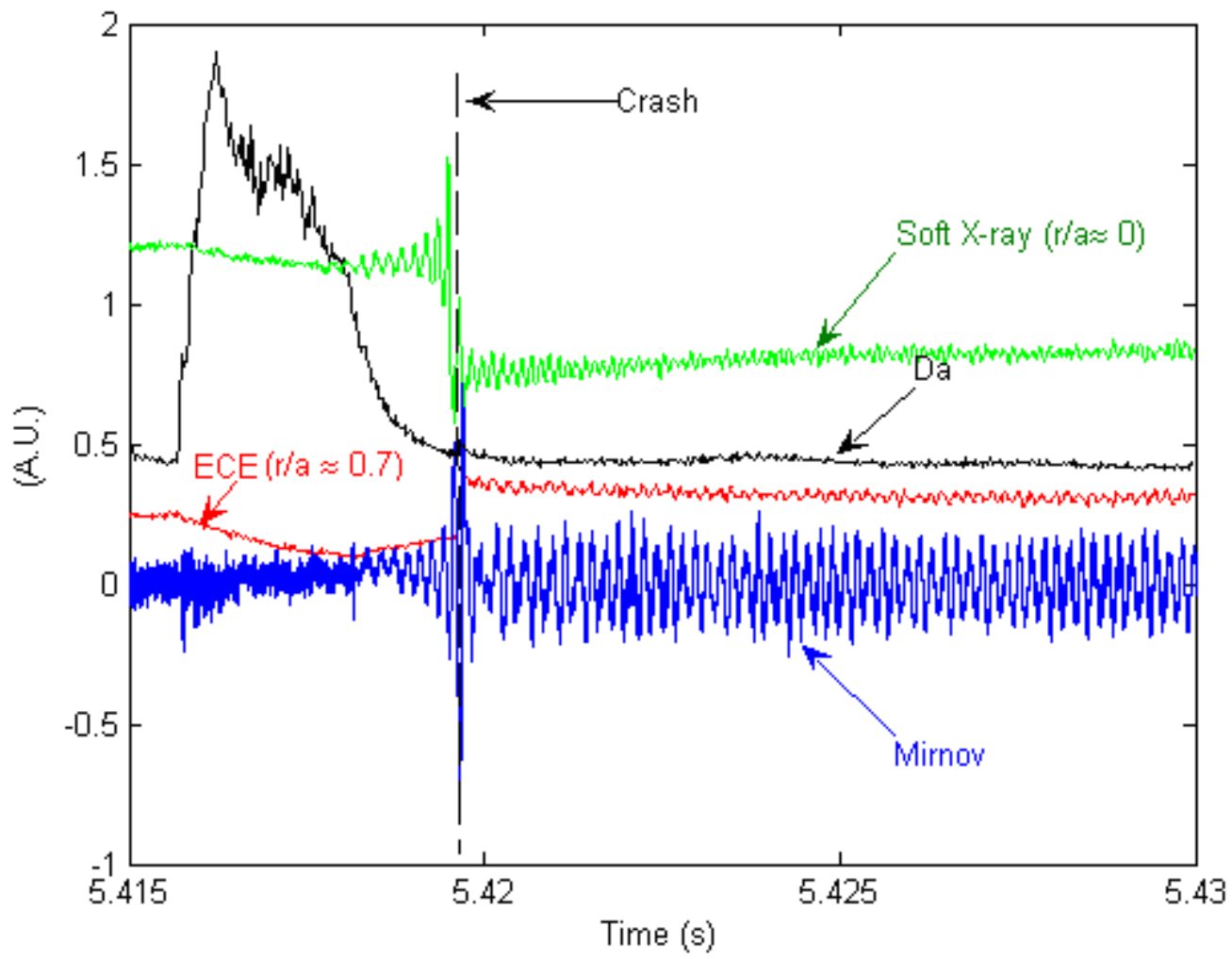

Figure 3 


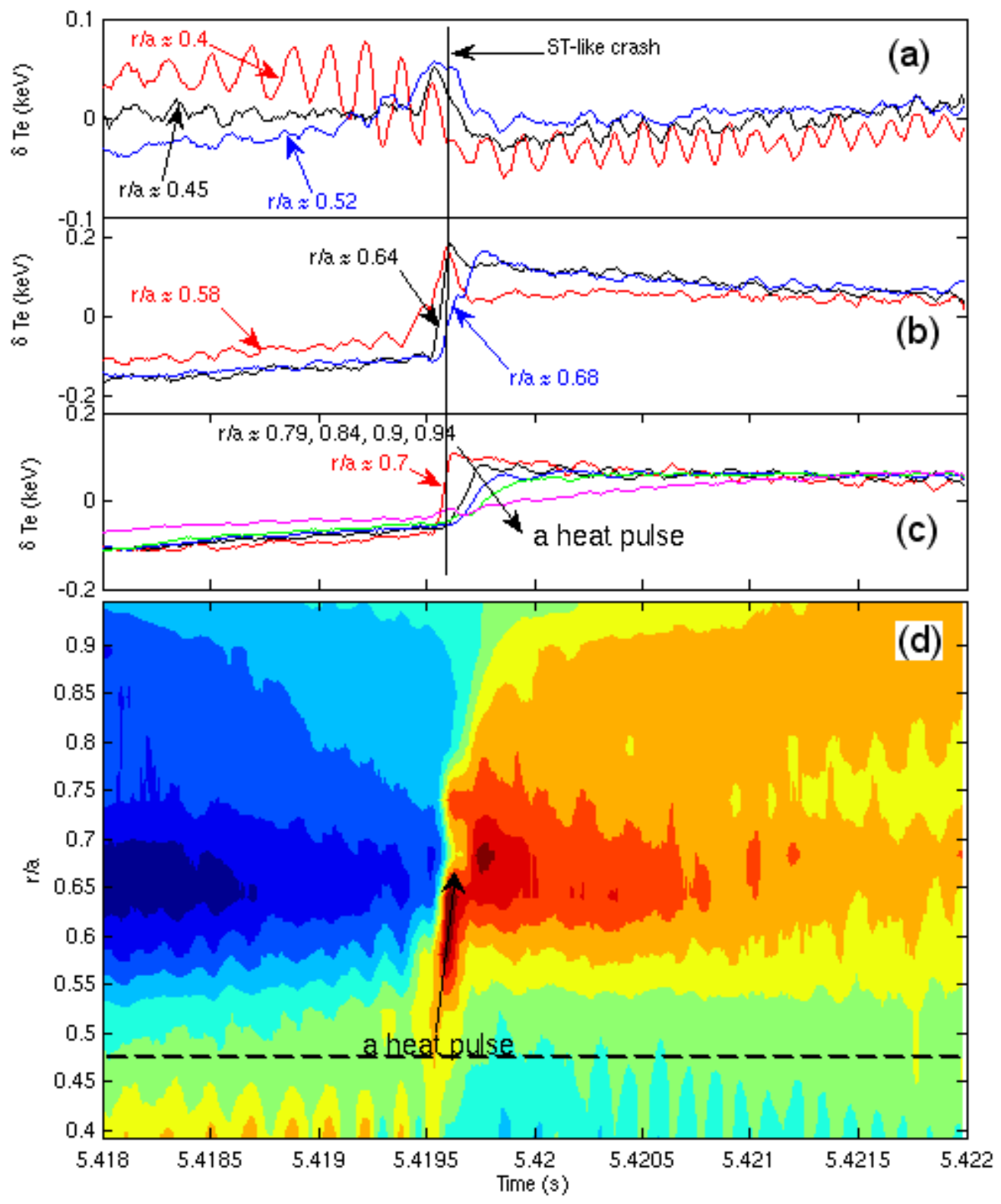

Figure 4 
(a)
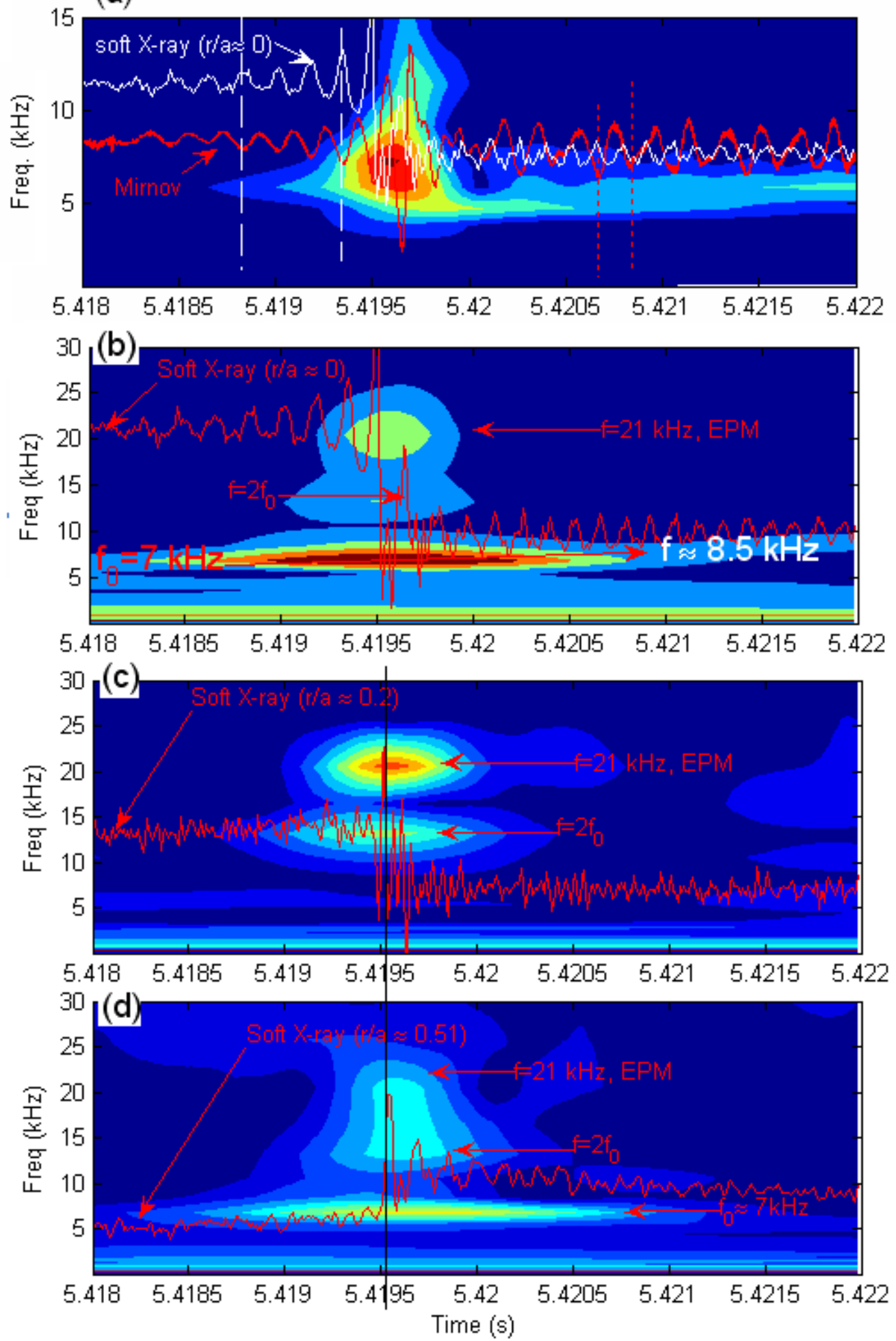

Figure 5 
(a)

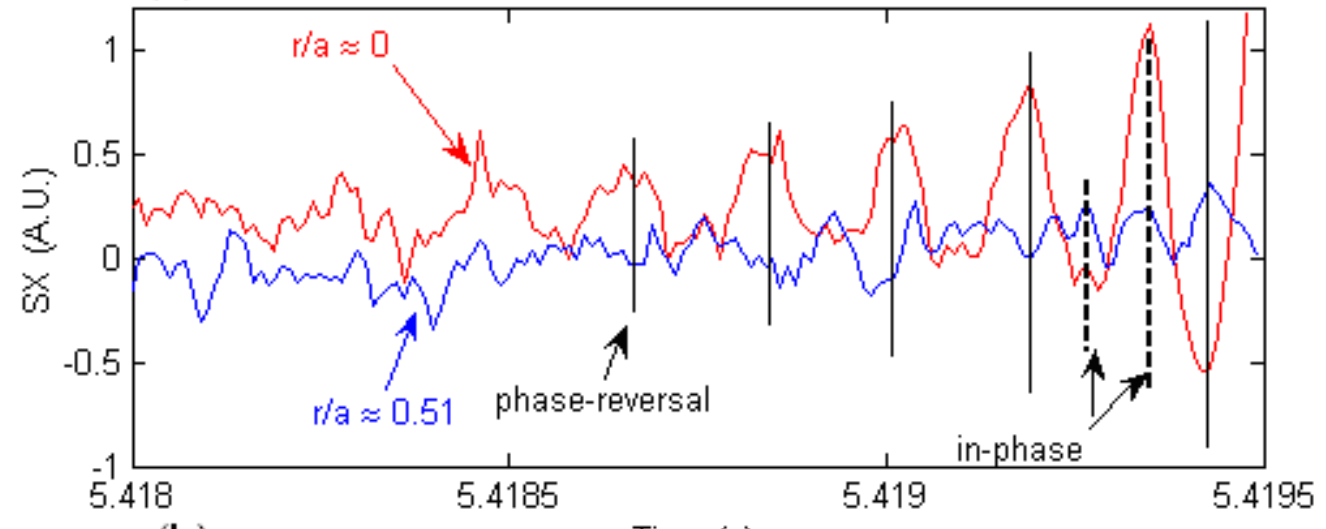

(b) Time (s)

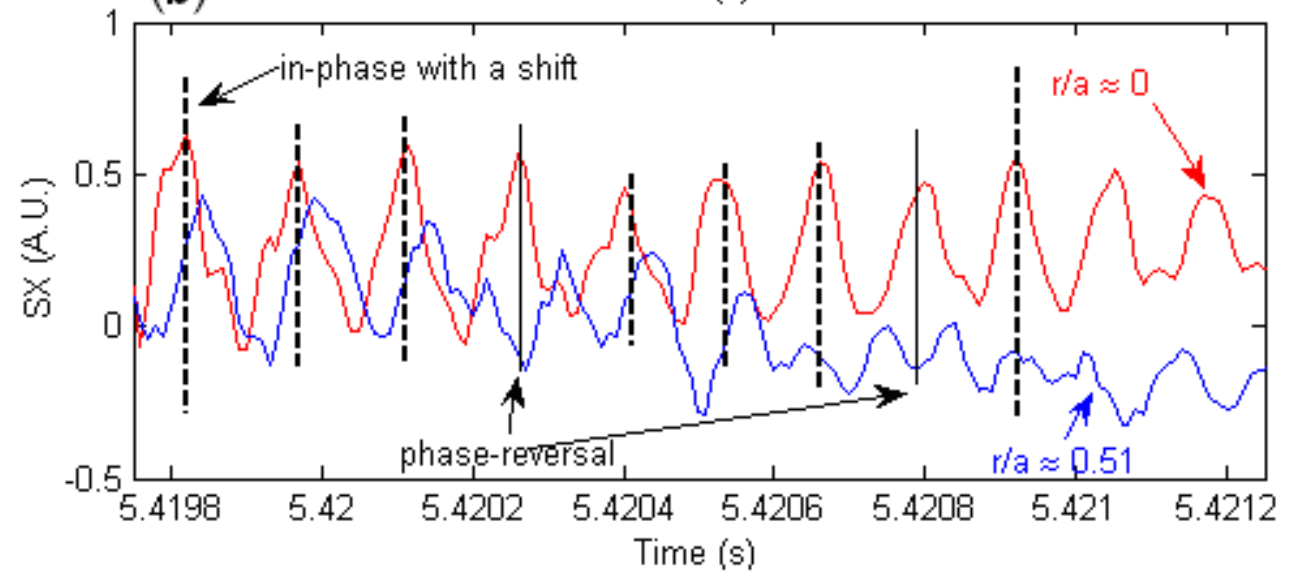

Figure 6 

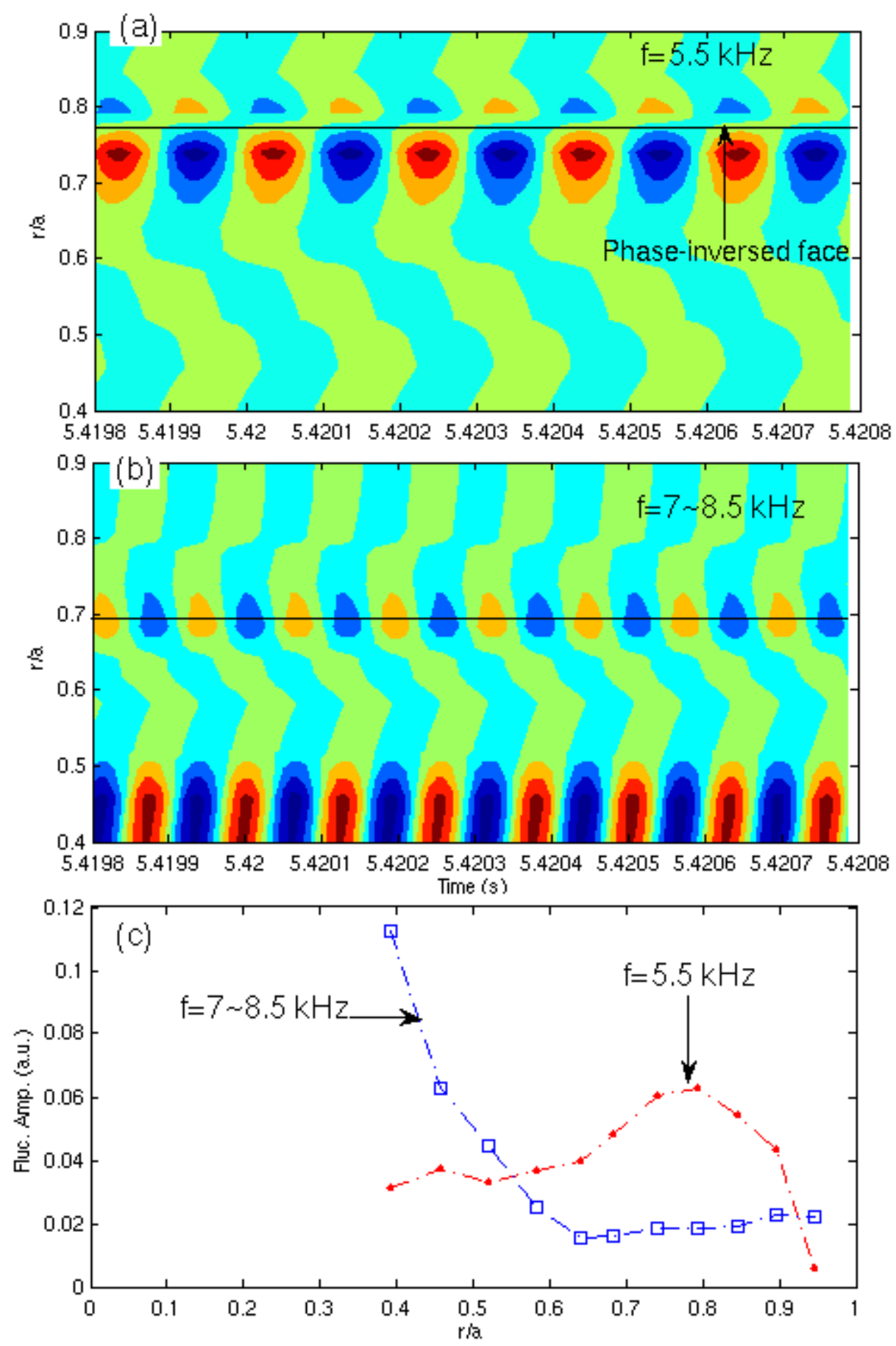

Figure 7 

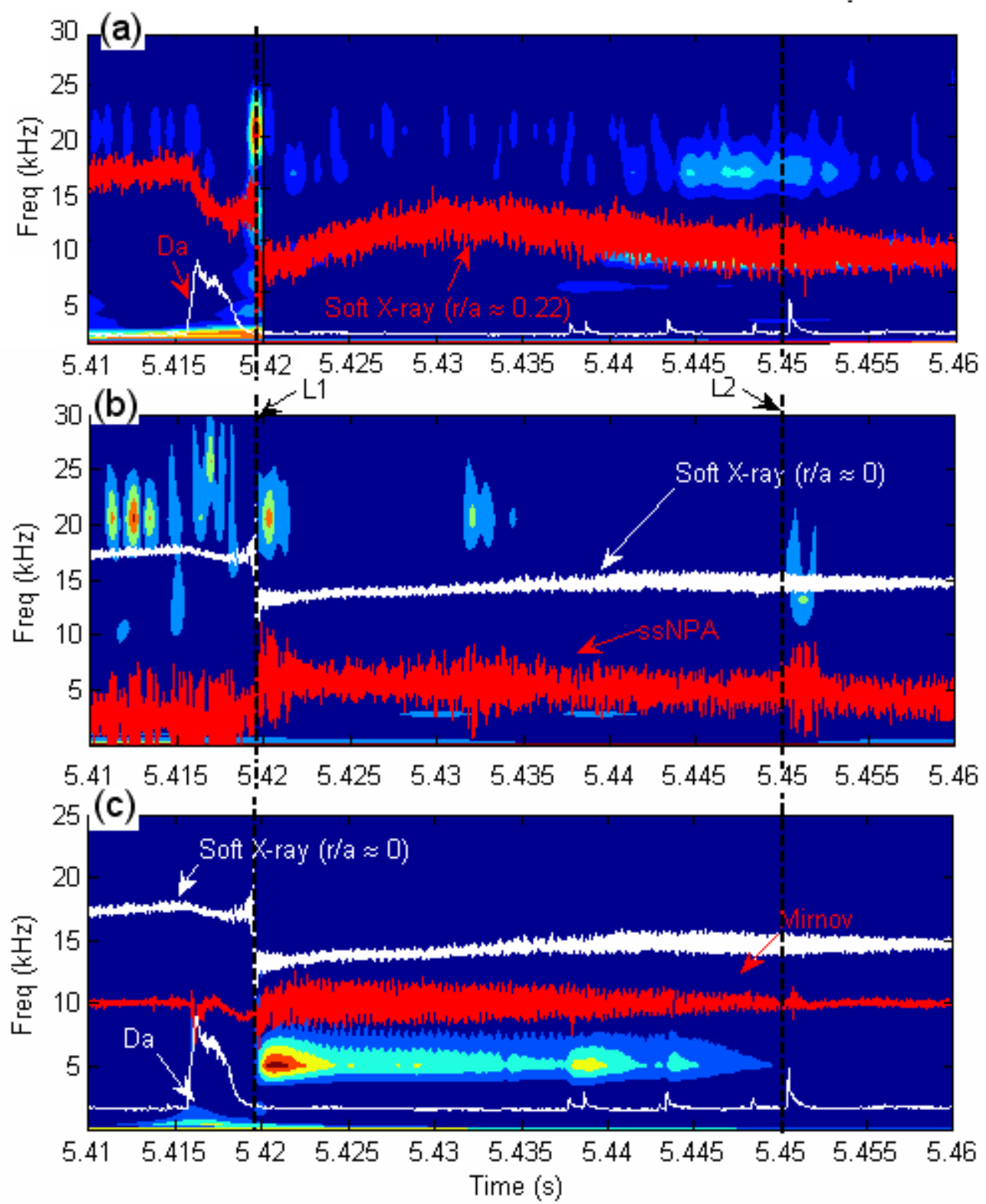

Figure 8 

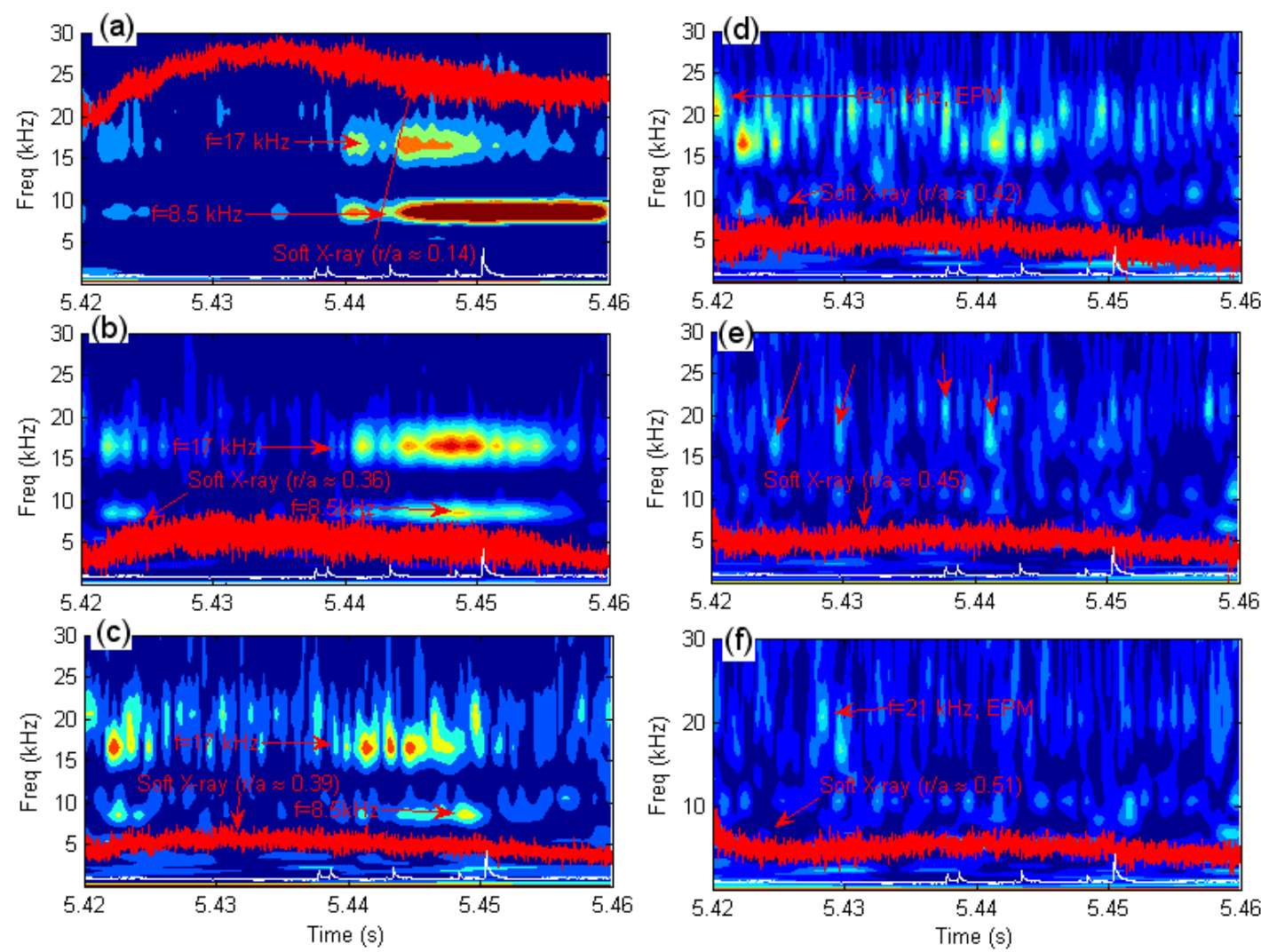

Figure 9 

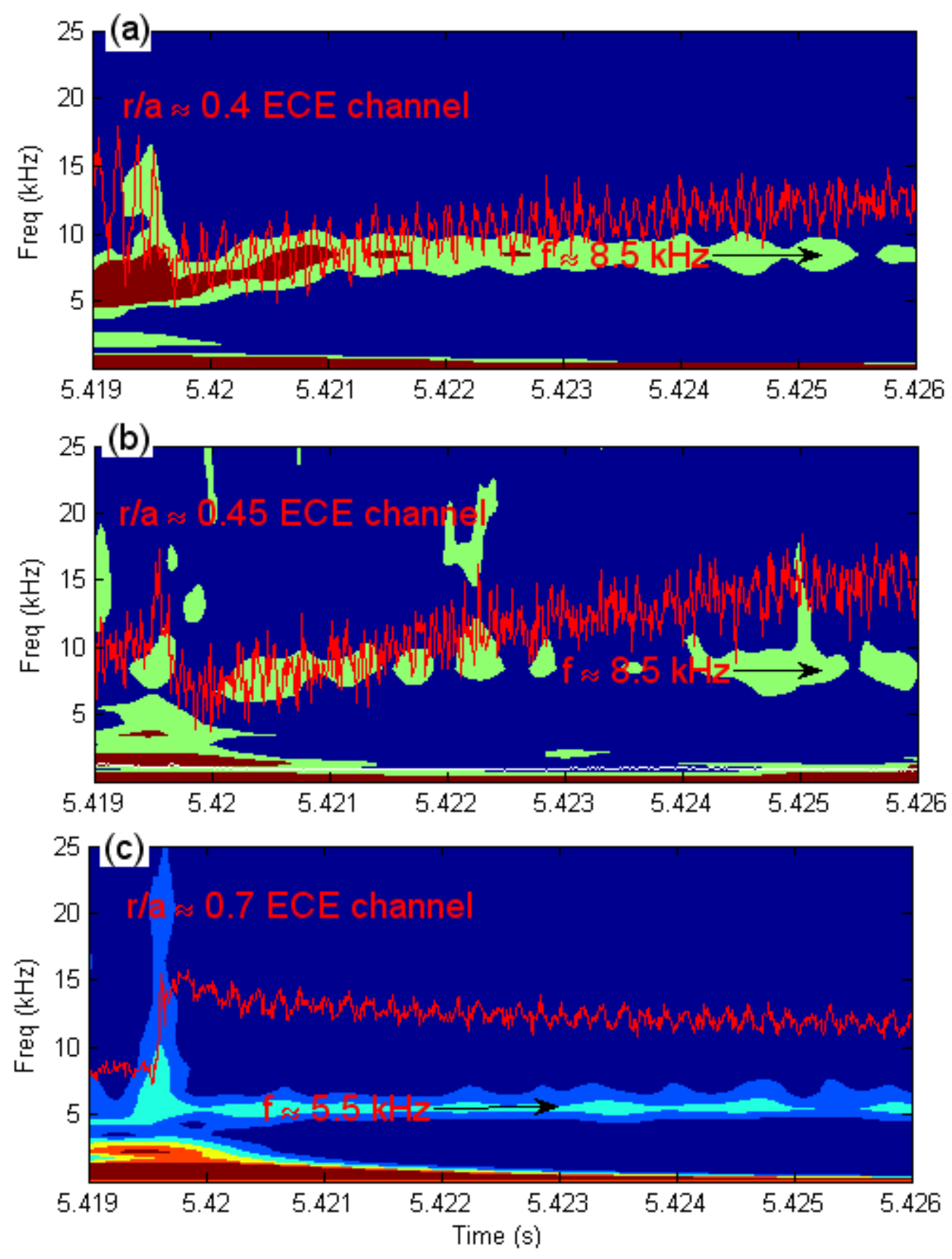

Figure 10 

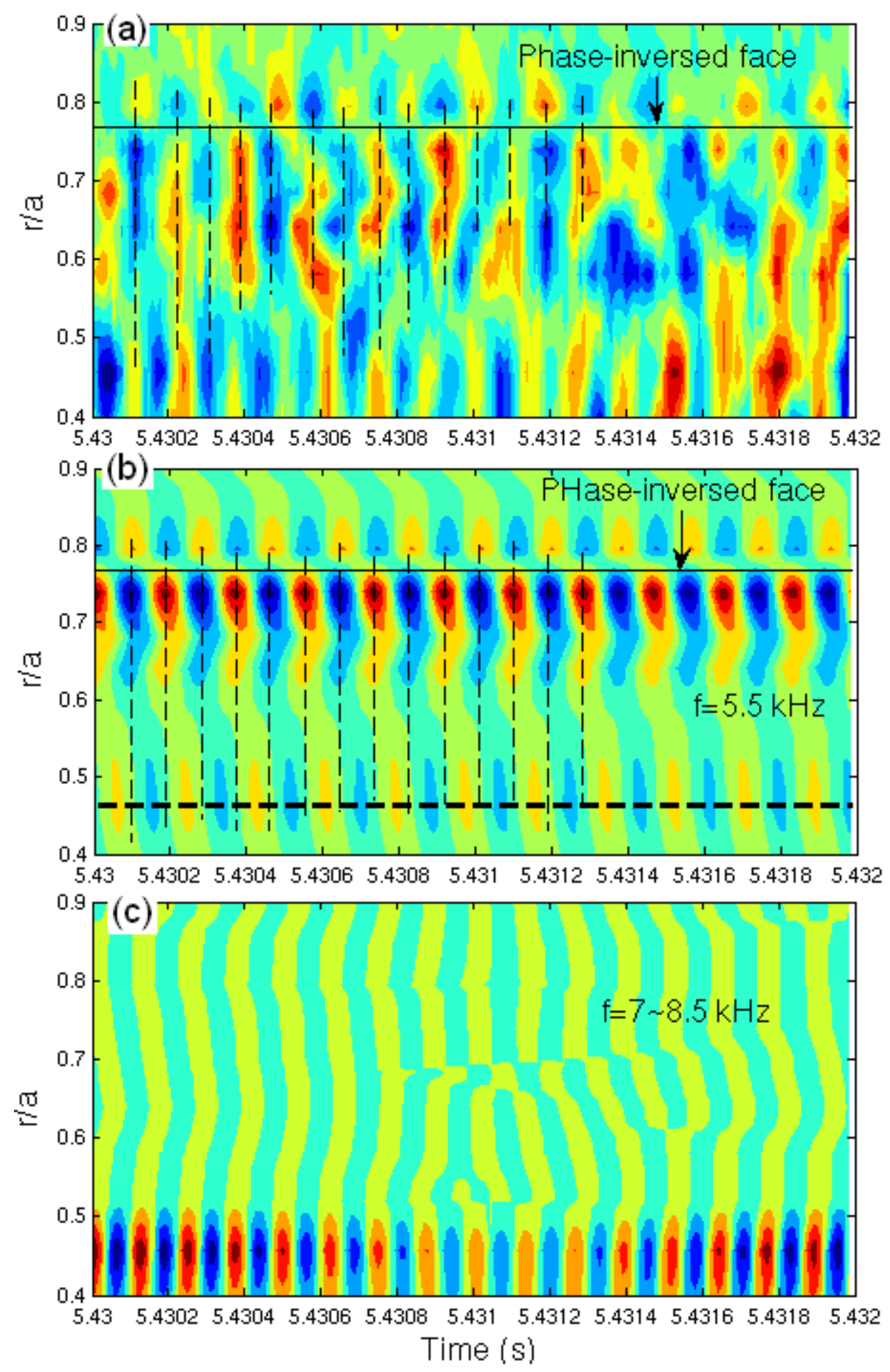

Figure 11 

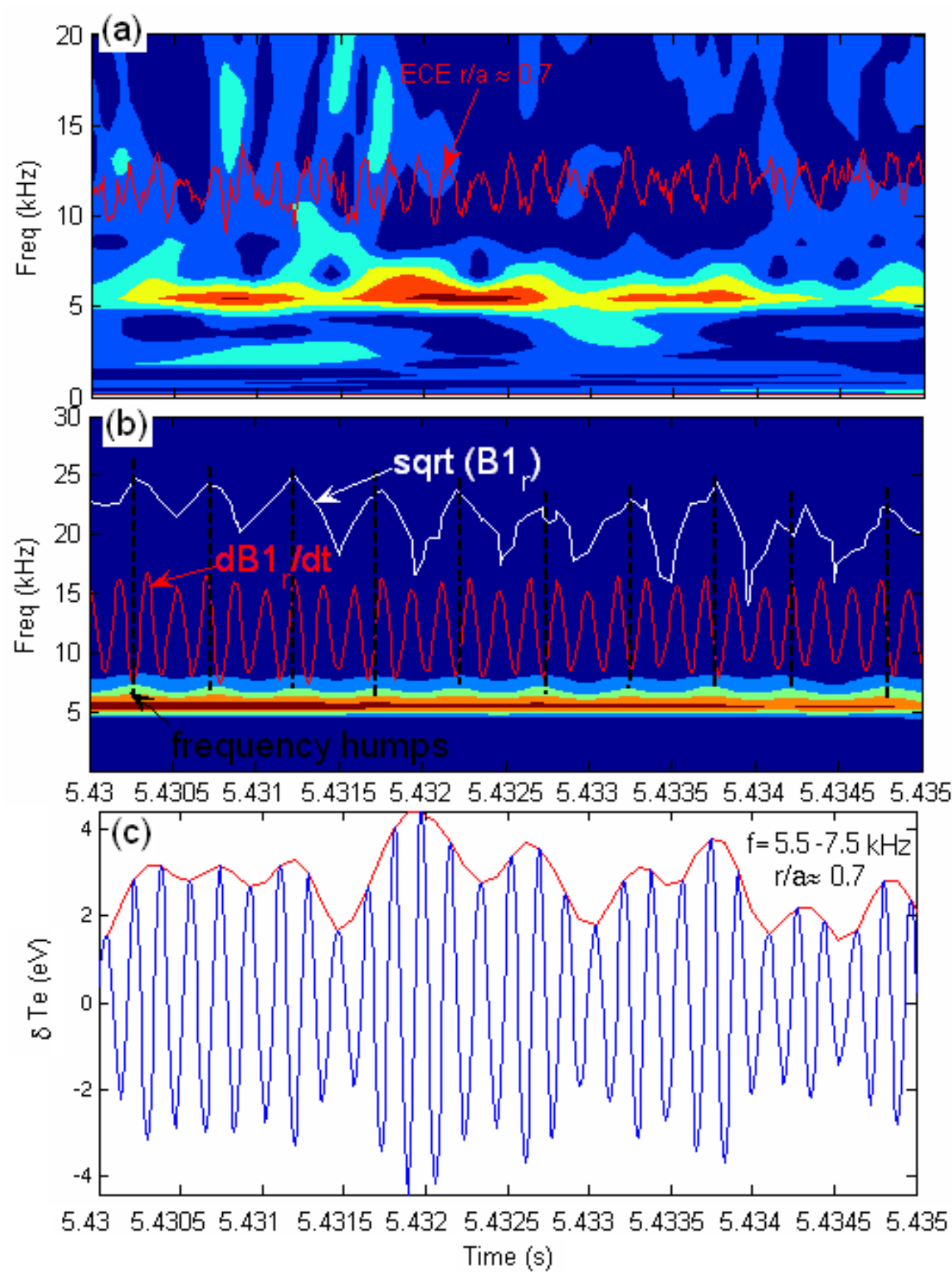

Figure 12 

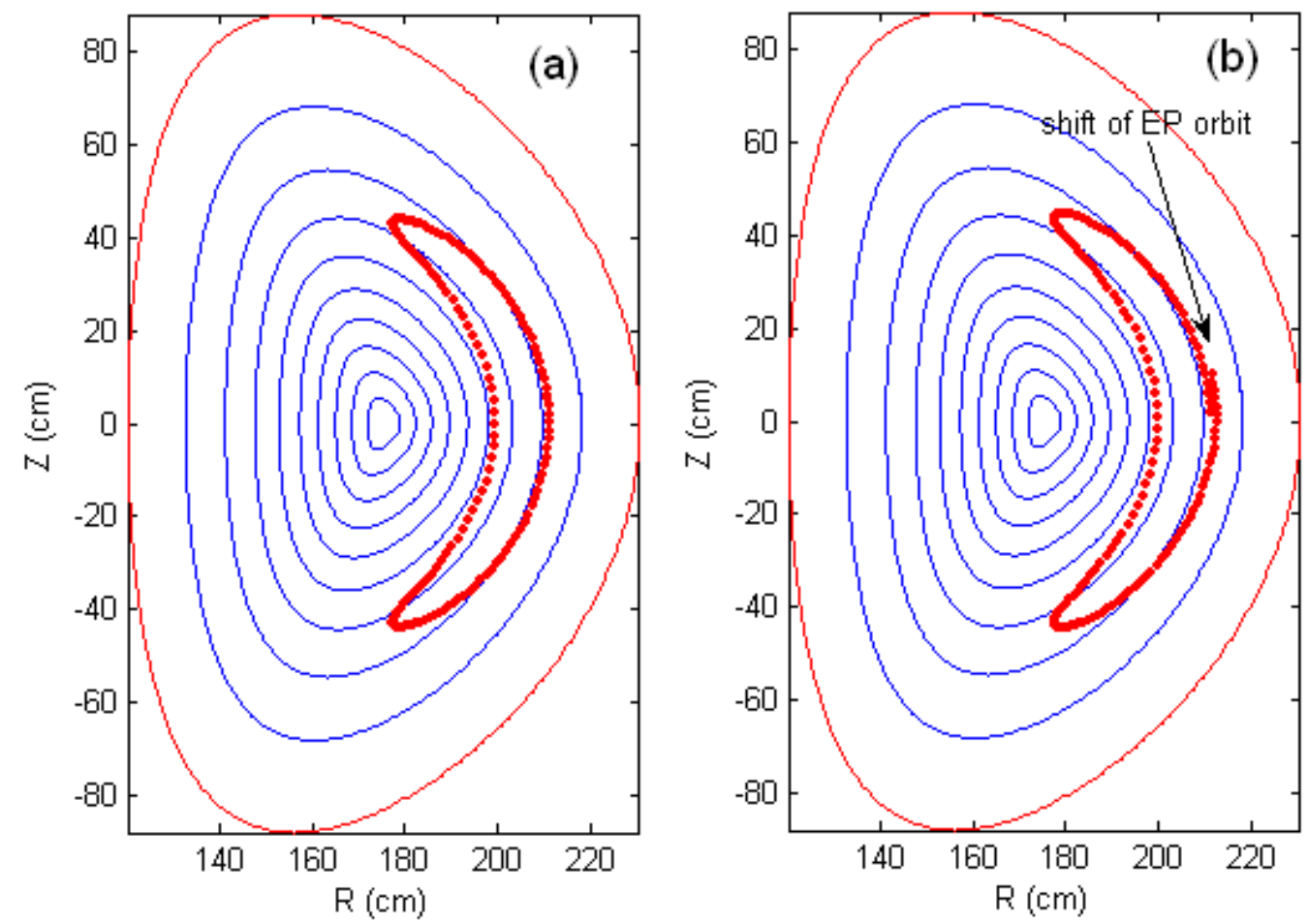

Figure 13 

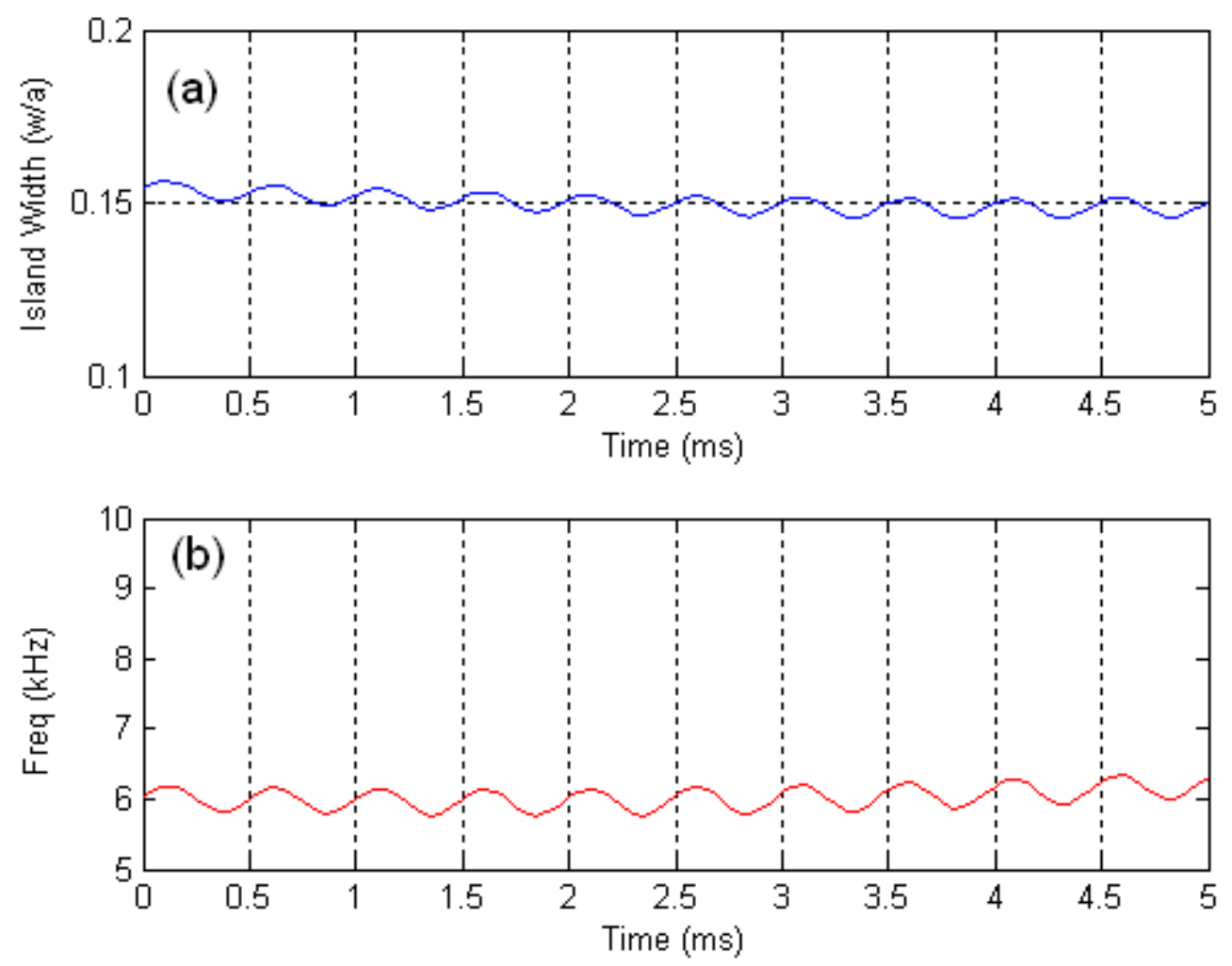

Figure 14 